\title{
Puericultura e educação em saúde - um relato de experiência
}

\section{Child care and health education - an experience report}

DOI: $10.46919 / \operatorname{archv} 1 \mathrm{n} 5-012$

Recebimento dos originais: 10/07/2020

Aceitação para publicação: 30/08/2020

\section{André Alexey Polidoro}

Mestre em Saúde e Gestão do Trabalho pela Universidade do Vale do Itajaí (UNIVALI).

Instituição: Universidade do Vale do Itajaí

Endereço: Rua Uruguai, 458 - Centro, Itajaí - SC, Brasil

E-mail: andre_alexey@hotmail.com

\section{Rodrigo Cechelero Bagatelli}

Mestre em Saúde e Gestão do Trabalho pela Universidade do Vale do Itajaí (UNIVALI). Instituição: UNIMED - PR

Endereço: Rua Affonso Pena, 837, Bairro Tarumã, Curitiba - PR, Brasil

E-mail: rodrigobagatelli@yahoo.com.br

\section{Aline Elias da Vinha Andrade Allegretti}

Residência em Medicina da Família e Comunidade pela Pontifícia Universidade Católica do Paraná. Instituição: Universidade Positivo Endereço: Rua Prof. Pedro Viriato Parigot de Souza, 5300, Ecoville, Curitiba - PR, Brasil

E-mail: alineevandrade@yahoo.com.br

\section{Juliana Schuster Semmer Krambeck}

Residência em Medicina da Família e Comunidade pela Pontifícia Universidade Católica do Paraná.

Instituição: Prefeitura do Município de Rio Negro - PR

Endereço: Rua Dr. Getulio Vargas, 130, Centro, Rio Negro - PR, Brasil

E-mail: julianamedfam@outlook.com

\section{Jacqueline Aedinet Pruner Polidoro}

Residência Multiprofissional em Atenção Básica pela Universidade do Vale do Itajaí (UNIVALI) Instituição: Prefeitura do Município de Porto Belo - SC Endereço: Avenida Governador Celso Ramos, 2500, Centro, Porto Belo - SC, Brasil E-mail: jackipruner@gmail.com

\section{RESUMO}

$\mathrm{O}$ atendimento de rotina às crianças saudáveis, também chamado de puericultura, é atualmente função executada pela equipe de Estratégia Saúde da Família (ESF), de forma multidisciplinar, nas unidades de saúde, fazendo com que todos os membros da equipe trabalham conjuntamente neste cuidado. A necessidade e a origem desta prática são alvos de debates antropológicos e da própria Medicina Baseada em Evidências, mas a puericultura faz parte atualmente das recomendações no Ministério da Saúde e é uma demanda da parte dos pais. Além disso, a necessidade de ensinar os pais o cuidado de saúde com os filhos faz parte dessa atividade. Esta necessidade aumenta a pressão sobre a já sobrecarregada equipe de ESF. Este artigo mostra a elaboração em equipe de roteiro multiprofissional para a avaliação da criança em diferentes idades, e constrói a atividade coletiva de puericultura como um encontro entre população e 
profissionais de saúde para troca de saberes, numa relação horizontal, com o objetivo de construção de autonomia, através da educação em saúde.

Palavras-chave: Educação em saúde, Puericultura, Unidade de saúde, Atenção Primária.

\begin{abstract}
Routine care for healthy children is currently a function performed by the Family Health Strategy (ESF) team, in a multidisciplinary way, at health units, making all team members work together in this care. The need and origin of this practice are targets of anthropological and Evidence-Based Medicine debates, but childcare is part of the Health Ministry recommendations and is a parent demand. In addition, the need to teach parents how to care for their children is part of this activity. This need increases the pressure on the already overworked ESF team. This article shows the team's elaboration of a multiprofessional script for evaluation of children at different ages, and it builds the collective childcare activity as a meeting between population and health professionals to exchange knowledge, by horizontal relationships, to building autonomy through health education.
\end{abstract}

Keywords: Health education, Child Care, Health Unit, Primary Care.

\title{
1 INTRODUÇÃO
}

$\mathrm{O}$ atendimento de crianças saudáveis ainda hoje é parte do escopo de práticas médicas ${ }^{1}$. Sua realização rotineira está pautada pela concepção de que identificar precocemente alterações no desenvolvimento é essencial, favorecendo avaliação específica, diagnóstico e intervenção terapêutica, com plano de acompanhamento para a criança e a família ${ }^{2}$. A esta atividade denominou-se puericultura, definida como “conjunto de técnicas empregadas para assegurar o perfeito desenvolvimento físico e mental da criança, desde o período de gestação até a idade de 4 ou 5 anos, e, por extensão, da gestação à puberdade"3. A literatura enfatiza ainda a continuidade dessas ações, a fim de atingir o objetivo: crianças e adultos saudáveis, felizes e com melhor qualidade de vida ${ }^{4}$.

A puericultura abrange não apenas a vigilância do desenvolvimento da criança e a busca de alterações ou patologias, mas também apresenta uma série de orientações em saúde, para prevenção de agravos $^{6}$. Este serviço ocupa cerca de $40 \%$ das agendas dos pediatras ${ }^{7}$, explicando uma parte dos motivos pelos quais, apesar da falta de evidências e de ausência de resultados positivos comprovados, manteve-se como atividade hegemônica ${ }^{8}$.

A crítica feita a esta atividade também está extensamente demonstrada em diversos artigos, pois a puericultura é alvo de debates nas áreas da saúde e também da sociologia, principalmente após a década de $60^{9}$. Nesta concepção, há uma proposta oculta, anterior à diminuição da mortalidade infantil, como o estabelecimento de um padrão comportamental, para as crianças e suas famílias, de cunho moralizante e orientado pelas classes dominantes ${ }^{10}$. 
Esta percepção é baseada na visão histórica da "preocupação" dos Estados com a "sobrevivência das crianças até a vida adulta" advinda principalmente da necessidade de exércitos, mão-de-obra e mercado ${ }^{11}$. Foucalt, em sua crítica, denomina os cuidados do Estado com suas populações como "o privilégio da infância e a medicalização da família”, e analisa ainda a disseminação, nesta época, da sugestão de que a vida deve seguir recomendações médicas.

A prática da puericultura e, principalmente, da monitorização mensal do crescimento também tem sido vítimas de duras críticas da própria Medicina Baseada em Evidências, baseado em revisões sistemáticas que mostram ausência de benefício da atividade e que, além disso, mostram capacidade de criar malefícios, ferindo inclusive um dos princípios da bioética clássica: “primum non nocere" ${ }^{\text {. Essas }}$ contradições presentes na puericultura estão presentes também no cotidiano do trabalho do profissional.

Assim, a partir deste entendimento inicial sobre a puericultura, é necessário localizarmos o atendimento às crianças na esfera organizacional do Sistema Único de Saúde (SUS). A avaliação do crescimento e do desenvolvimento infantil acontece na Atenção Básica, definida pelo Ministério da Saúde (MS) como "um conjunto de ações, de caráter individual ou coletivo, situadas no primeiro nível de atenção dos sistemas de Saúde, voltadas para a promoção da saúde, prevenção dos agravos, o tratamento e a reabilitação" ${ }^{2}$. O Ministério da Saúde ressalta ainda a função da Atenção Primária à Saúde (APS) no cuidado da criança:

\begin{abstract}
“até a criança completar 2 anos, o objetivo é um acompanhamento cuidadoso do crescimento e do desenvolvimento da criança pela equipe de saúde (inclusive com busca de faltosos), com um olhar biopsicossocial não só para a criança, mas também para as condições do contexto de saúde e de vida de sua mãe e família, inclusive com as articulações intersetoriais, no território, necessárias para o projeto terapêutico de cada criança/família"13.
\end{abstract}

Ainda se faz necessário entender o contexto de quem fará o atendimento a esta criança. Há diversos debates sobre quem é o profissional indicado para a avaliação do desenvolvimento da criança, que fogem do objetivo deste relato ${ }^{14}$. Porém, em 1994, foi proposta uma nova forma de organização da atenção básica, através do Programa de Saúde da Família (PSF) ${ }^{15}$, implantado em 2006 como estratégia nacional, a Estratégia de Saúde da Família $(E S F)^{16}$. Neste modelo, a atenção à criança é realizada pelo médico de família, o enfermeiro, o auxiliar de enfermagem e o agente comunitário de saúde, onde todos participam do cuidado ${ }^{17}$. A proposta desta estratégia era ampliar a cobertura dos serviços de saúde, rediscutir as ações educativas e enfatizar a integralidade da assistência ${ }^{36}$.

Entretanto, a equipe de saúde da família depara-se com várias dificuldades. A pressão pelo número excessivo de consultas recomendadas por vários protocolos ${ }^{14,18}$, o grande número de condutas e procedimentos sem caráter científico (mas que as vezes causa satisfação aos pais) ${ }^{14,19}$, a necessidade de 
acompanhar o desenvolvimento da criança ${ }^{13}$, a prevenção quaternária, o risco de tentar "normatizar" as $\operatorname{crianças~}^{20}$, a necessidade de educação em saúde ${ }^{21}$, não esquecendo que a equipe ainda é responsável por todas as outras demandas sua área de abrangência, de todas as faixas etárias e inclusive aquelas em relação às crianças que necessitam de consulta em caráter de urgência ${ }^{22}$.

A descrição da experiência a seguir objetiva a operacionalização da, mesmo contraditória, puericultura, apoiando sua prática em educação em saúde e na avaliação objetiva individual da criança, a partir de um roteiro construído pela própria equipe.

\section{METODOLOGIA}

Relato de experiência com investigação bibliográfica. Metodologia descritiva, indutiva e analítica.

Foi realizada uma pesquisa bibliográfica nas plataformas: Portal de Evidencias/Ministério da Saúde e ProQuest-Saúde da Família, com as palavras chave: "puericultura", "avaliação da criança", "desenvolvimento", "rotina da criança". Também foi pesquisado nas plataformas Pubmed e Cochrane e no Jornal American Academy of Family Pishician, com as palavras: "childcare", "child development", "grouth monitoring". Os artigos foram selecionados pela percepção de relação com o tema, pelo resumo ou introdução.

\section{DESCRIÇÃO DA EXPERIÊNCIA}

A experiência aconteceu na área 2 de uma unidade de saúde, de ESF, localizada na periferia de Curitiba. $\mathrm{O}$ atendimento médico desta área era feita por residentes de Medicina de Família e Comunidade da Pontifícia Universidade Católica do Paraná. A população assistida era de 3900 habitantes. Na prática, o residente atendia durante 30 horas semanalmente (excluindo tempo de visitas, reuniões de equipe e preceptoria). Tendo em vista a programação da agenda com oferta de cerca de $40 \%$ da agenda para demanda espontânea, restavam ao residente semanalmente 18 horas para as consultas de rotina, agendadas e as de retorno.

A área citada possuía 23 crianças menores de 1 ano e 26 crianças com idade entre 1 e 2 anos. A recomendação geral no município era que as crianças fossem acompanhadas mensalmente pela equipe da ESF até completar 01 ano de idade, podendo-se aumentar ou diminuir a frequência dependendo da necessidade percebida pelos profissionais de saúde. No segundo ano de vida, a recomendação era de espaçar a frequência para visitas a cada trimestre, também dependendo da observação subjetiva da equipe. Como o contexto social da região é de risco social, praticamente todas as crianças tinham alguma vulnerabilidade a ser destacada na sua avaliação e, tecnicamente, encontravam-se em risco aumentado de adoecimento. 
Desta forma, em uma época em que os programas verticais eram diretivos, foi realizado um cálculo para inclusão de todas as crianças na agenda médica, causando, porém, grande impacto na agenda médica, sobrecarregando mais ainda a agenda com consultas programáticas e prejudicando a oferta de consultas, e até mesmo a universalidade.

A pressão por atendimento na Unidade de Saúde era um problema crônico e uma das formas que a equipe entendeu ser possível para garantir o acompanhamento das crianças e proporcionar um momento para discutir com os pais assuntos de interesse deles, além de vencer a imposição de realizar as consultas mensais das crianças, foi organizar a agenda incluindo atividade coletiva mensal de puericultura, apelidada de Programa da Criança. Neste momento, com duração de 2 horas, após conversa inicial de curta duração com os pais da criança, as mesmas eram avaliadas ora por médico, ora por enfermeiro, com auxílio dos auxiliares de enfermagem e dos agentes comunitários de saúde. A divisão entre médico e enfermeira era a depender da idade da criança, havendo, por exemplo, para as crianças de baixo risco, 8 avaliações médicas nos 2 primeiros anos.

Para a avaliação das crianças, a equipe elaborou conjuntamente um roteiro, com tópicos a serem observados na criança, de maneira objetiva. Ao contrário de tentar simplificar a infância ou reduzi-la a doenças, o roteiro foi útil apenas como ferramenta para auxiliar os membros da equipe a ter um olhar direto e específico para cada criança e aos aspectos relacionados às respectivas idades, visto o grande número de informações nesta área, com níveis variados de evidência, além de favorecer a lembrança de múltiplos aspectos relacionados à heterogeneidade de idades presentes no programa.

Simplificando e facilitando a avaliação clínica através do roteiro, foi feito melhor aproveitamento do tempo, possibilitando à equipe debruçar-se sobre aspectos importantes no acompanhamento das crianças, buscando superar o caráter padronizador da puericultura através de prática dialógica e participativa.

Foram formados assim grupos de educação em saúde, com a participação de toda equipe e dos pais das crianças. Porém, educação em saúde, bem como qualquer outra prática dentro do complexo campo da saúde, pode ser entendida de diversas formas a depender de estilo de pensamento adotado ${ }^{23}$.

A maneira hegemônica de se pensar a educação a saúde e, por isso, de realizar ações, está relacionada à mudança do estilo de vida para um estilo mais saudável, cabendo ao indivíduo reduzir os riscos autoimpostos. Porém, em oposição, sabe-se que, em ambientes desfavoráveis, responsabilizar a pessoa e culpá-la por não adotar um determinado estilo de vida pode levar ao sentimento de incapacidade, que causa passividade, que por sua vez contribui para incapacidade de mudança ${ }^{24}$. Esse processo denomina-se, na literatura, culpabilização da vítima, reduzindo algo abrangente e de complexo nexo causal à visão individual e à causalidade linear ${ }^{25}$. 
Uma ação de educação em saúde baseada no modelo de Promoção à Saúde Behaviorista traria estratégias educacionais vinculadas a uma relação pedagógica vertical, em que o saber técnico é superior à autonomia do sujeito, e traria informações às pessoas sobre atitudes saudáveis, de forma a influenciar hábitos e comportamentos ${ }^{26}$.

A construção do conhecimento com a pessoa, como buscado nas reuniões realizadas, não nega a possibilidade dessa emancipação individual, mas considera ainda o contexto político e histórico das pessoas envolvidas, pensando a saúde como um bem comum e buscando o desenvolvimento da capacidade individual e comunitária (Nova Promoção da Saúde) ${ }^{28}$. Além de uma compreensão ampliada do processo saúde-doença, atua na transformação na equidade, reflexão crítica e fortalecimento comunitário $^{29}$, sem deixar de lado a observação da necessidade de políticas públicas que favoreçam esse processo.

Diferente do que era feito anteriormente, a equipe empenhou-se em trabalhar com as pessoas, e não pelas pessoas, como orienta a Declaração de Jacarta ${ }^{27}$. Houve um deslocamento do foco na doença para a saúde e os processos de decisão foram atrelados às subjetividades dos atores nos seus espaços do cotidiano. Essa valorização da participação social está na base desta perspectiva de promoção à saúde ${ }^{30}$.

O grupo de puericultura não era um ambiente para repassar conhecimentos, mas de construção coletiva do mesmo e, dessa maneira, a prática pedagógica pôde tornar-se transformadora, dialógica e pautada no exercício da consciência crítica reflexiva ${ }^{31}$.

Voltando ao campo das ideias, temos que como consequências dos dois modelos de promoção à saúde demonstrados, pode-se notar duas tendências na área da educação em saúde ${ }^{39}$. A primeira, denominada educação sanitária, volta-se ao modelo biologicista e, consequentemente, leva a uma prática preventivista. Esta concepção é marcada pelo estilo autoritário (pedagogia vertical), na qual o educador é o conhecedor da verdade científica e a transmite a população ${ }^{32}$. Assim, acarreta a responsabilização individual pela sua saúde e a modificação do comportamento. Ao poder público, caberia a responsabilidade de internalizar comportamentos adequados nas pessoas ${ }^{26}$.

A segunda tendência recebe o nome de educação em saúde propriamente dita. Diferencia-se pela busca de formar cidadãos críticos e transformadores da sociedade, tendo como referência o trabalho de Paulo Freire ${ }^{32}$. O diálogo, na percepção deste, é um encontro entre homens mediados pelo mundo ${ }^{37} \mathrm{e}$ inicia-se na busca do tema, quando o educador-educando se pergunta em torno do que vai dialogar como educando-educador ${ }^{38}$. Trata-se de uma relação biunívoca, com troca e construção de novos saberes.

Dessa maneira, as atividades coletivas em puericultura, tentavam colocar em prática o significado de promoção à saúde, presente parcialmente na Carta de Ottawa, 1986: "Promoção da saúde é o nome dado ao processo de capacitação da comunidade para atuar na melhoria de sua qualidade de vida e saúde, 
incluindo uma maior participação no controle deste processo" ${ }^{40}$; e complementado na Política Nacional de Promoção à Saúde, que destaca a necessidade de políticas públicas favoráveis à saúde e à vida, estimulando cidadãos a se envolverem na sua elaboração e implementação ${ }^{45}$. Apesar do tempo limitado para realização de todas as tarefas almejadas, considerou-se de maior importância o trabalho de reforçar a autonomia do sujeito, conceito que está ligado à emancipação dos sujeitos e à participação popular ${ }^{33}$, visando, ainda, a promoção à cidadania e à qualidade de vida ${ }^{36}$. Pra Fleury-Teixeira, et al, 2008, a autonomia é a categoria central no conceito de promoção à saúde, atingido neste trabalho que integrou mães e pais de crianças e profissionais da atenção básica ${ }^{46}$.

\section{CONSIDERAÇÕES FINAIS}

Após cerca de 6 meses de experiência, concebemos que a busca por exercer uma a educação em saúde de modo a torná-la um instrumento de promoção de saúde de modo participativo, dialógico e emancipatório não se tratou apenas de um resultado, mas de um processo árduo para valorizar a autonomia da população e sua corresponsabilidade no processo saúde-doença ${ }^{34}$. Processo porque envolve um movimento contínuo e que considera a questão sócio-histórica. Árduo pela grande limitação dos profissionais envolvidos no processo, pela dificuldade de se desvincularem da lógica hegemônica do modelo biomédico ${ }^{35}$, afinal, a formação médica atual não prepara o profissional para o trabalho interdisciplinar, nem para respeitar autonomia das pessoas, ou para o processo de fortalecimento comunitário $^{41}$.

Na formação em saúde, pouco aprendemos também sobre o "falar contigo", em oposição ao "falar a ti’"43. Porém, o trabalho com as pessoas (e não para as pessoas) gera, como já dizia Paulo Freire, aprendizado no educador-educando e no educando-educador, possibilitando um movimento em direção à construção do conhecimento e alterando a percepção da equipe quanto ao próprio processo de aproximação da comunidade. Também foi possível observar que esta prática, aumentou a coesão da equipe, o trabalho interdisciplinar e a satisfação e dos pais com o programa, percebido também pelo aumento da assiduidade.

A partir dos olhos da gestão, essas atividades permitiram à equipe conhecer as crianças da área e manter o número esperado de visitas das mesmas à unidade, seguindo a concepção de puericultura inicialmente descrita.

Estes dois resultados associados levam a uma conclusão interessante, visto que, nesta experiência, associou-se uma atividade baseada em pressupostos biomédicos a uma atuação do campo da saúde coletiva. Normalmente, são vistas como duas macrotendências que disputam o controle das práticas em saúde (o modelo biomédico e o modelo da saúde coletiva ${ }^{26}$, porém a visão integral do processo saúde- 
doença incorpora elementos das concepções biologicista, social e higienista / preventivista ${ }^{42}$, não fazendo disso uma contradição, mas sim um dos aspectos da integralidade.

Experiências como estas levam ainda à reflexão sobre as várias formas de se promover saúde, diferentes daquelas propagadas pelo atual modelo hegemônico, fortalecendo a visão integral de saúde e a necessidade de reformas nos modelos atuais de educação aos profissionais de saúde.

\section{REFERÊNCIAS}

1. Blank, D. Acompanhamento de Saúde da Criança. In: Duncan, B.B.; Schimidt, M. I.; Giugliani, E. R. J. Medicina Ambulatorial: Condutas de Atenção Primária Baseadas em Evidência. Artmed, 2013. 20:170-80.

2. Council on Children With Disabilities, Section on Developmental Behavioral Pediatrics, Bright Futures Steering Committee and Medical Home Initiatives for Children With Special Needs Project Advisory Committee Identifying Infants and Young Children With Developmental Disorders in the Medical Home: An Algorithm for Developmental Surveillance and Screening Pediatrics 2006;118;405. DOI: 10.1542/peds.2006-1231

3. Ferreira ABH. Novo Aurélio Século XXI - O Dicionário da Língua Portuguesa. Rio de Janeiro: Nova Fronteira; 1999.

4. Schor, E.L. Rethinking well-child care. Pediatrics. 2004;114:210-6.

5. Ricco RG, Santoro JR, Almeida CA, Del Ciampo LA. Atenção à saúde da criança e puericultura. In: Ricco RG, Del Ciampo LA, Almeida CA. Puericultura: princípios e p'ráticas: atenção integral à saúde. São Paulo: Atheneu; 2000. p. 1-4.

6. MARCONDES, E. Ser puericultor. In: ISSLER, H.; LEONE, C.; MARCONDES, E. Pediatria na atenção primária. São Paulo: Sarvier, 1999. p.33-48.

7. Hoekelman RA. Well-child visits revisited. Am J Dis Child. 1983;137:17-20

8. Dinkevich E, Hupert J, Moyer VA. Evidence based well child care. BMJ 2001;323:846-9.

9. Bonilha, L.R.C.M.; Rivorêdo, C.R.S.F. Puericultura: duas concepções distintas. J Pediatr (Rio J). 2005;81(1):7-13

10. Boltanski, L. Puericultura y moral de clase. Barcelona: Laia; 1974.

11. Foucault M. Microfísica do poder. Rio de Janeiro: Graal; 1986.

12. BRASIL. Ministério da Saúde. Programas Saúde da Família e Agentes Comunitários de Saúde: atividades 1999. Brasília, 2000.

13. Brasil. Ministério da Saúde. Secretaria de Atenção à Saúde. Departamento de Atenção Básica. Saúde da criança : crescimento e desenvolvimento / Ministério da Saúde. Secretaria de Atenção à Saúde. Departamento de Atenção Básica. - Brasília : Ministério da Saúde, 2012. 272 p.: il. (Cadernos de Atenção Básica, no 33)

14. Blank, D. A puericultura hoje: um enfoque apoiado em evidências. J Pediatr (Rio J) 2003;79(Supl.1):S13-S22.

15. A Implantação da Unidade de Saúde da Família/Milton Menezes da Costa Neto, org. _Brasília: Ministério da Saúde; Secretaria de Políticas de Saúde, Departamento de Atenção Básica, 2000.

16. Brasil. Ministério da Saúde. Portaria n. 648, 298 de março de 2006. Aprova a Política Nacional de Atenção Básica, estabelecendo a revisão de diretrizes e normas para a organização da atenção básica para o Programa de Saúde da Família (PSF) e o Programa Agentes Comunitários de Saúde (PACS). Diário Oficial da União. 2006;seção 1:21. 
17. Sucupira, A.C.S.L. Saúde da Criança. In: Gusso, G.; LOPES, J.M.C. Tratado de Medicina de Família e Comunidade: princípios, formação e prática. Porto Alegre: Artmed 2012.

18. Green M, Palfrey JS. Bright Futures: Guidelines for Health Supervision of Infants, Children, and Adolescents. $2^{\mathrm{a}}$ ed. Arlington, VA: National Center for Education in Maternal and Child Health; 2002.

19. Brosco JP. Weight charts and well-child care: how the pediatrician became the expert in child health. Arch Pediatr Adolesc Med 2001;155:1385-9.

20. Novaes, H.M.D. A puericultura em questão. In: Mota A.; Schraiber, L.B. Infância e Saúde: perspectivas históricas. São Paulo: Hucitec; 2009.

21. Busey S, Schum TR, Meurer JR. Parental perceptions of well-child care visits in an inner-city clinic. Arch Pediatr Adolesc Med 2002;56:62-7.

22. Gusso, G.; Poli Neto, P. Gestão da Clínica. In: In: Gusso, G.; LOPES, J.M.C. Tratado de Medicina de Família e Comunidade: princípios, formação e prática. Porto Alegre: Artmed 2012

23. Fleck, L. (2010). Gênese e desenvolvimento de um fato científico (Otto \& Camilo de Oliveira, trads.). Fabrefactum (Trabalho original em alemão, 1935).

24. Figueira, T. R., Ferreira, E. F., Schall, V. T., \& Modena, C. M.(2009). Percepções e ações de mulheres em relação à prevenção e promoção da saúde na atenção básica. Rev.Saúde Pública, 43(6), 937-943.

25. Carvalho, S. R. (2004). As contradições da promoção à saúde em relação à produção de sujeitos e a mudança social. Ciência \& Saúde Coletiva, 9(3), 669-678.

26. Silva-Arioli, I.G.; Schneider, D.R.; Barbosa, T.M.; Da Ros, M.A. Promoção e Educação em Saúde: uma Análise Epistemológica. Psicologia, Ciência e Profissão, 2013, 33(3), 672-687.

27. The Jakarta Declaration on Leading Health Promotion into the $21^{\text {st }}$ Century. The 4th International Conference on Health Promotion;1997 Jul 21-5; Jakarta, Indonesia. Disponível em: http://www.who.int/hpr/archive/docs/jakarta/english.html

28. Carvalho, S. R. (2010). Saúde coletiva e promoção da saúde: sujeito e mudança (3a ed.). São Paulo: Hucitec.

29. Rabello, L. S. (2006). Promoção da saúde: desafio ou adaptação? A construção social do conceito, de Alma-Ata aos dias atuais, no Brasil e no Canadá. (Tese de Doutorado). Universidade de Brasília, Brasília, DF.

30. Czeresnia, D. (2009). O conceito de saúde e a diferença entre prevenção e promoção. In D. Czeresnia \& C. M. Freitas (Orgs.), Promoção da saúde: conceitos, reflexões, tendências. Rio de Janeiro: Ed. Fiocruz.

31. Buss, P. M. (2009). Uma introdução ao conceito de promoção da saúde. In D. Czeresnia \& C. M. Freitas (Orgs.), Promoção da saúde: conceitos, reflexões, tendências (2a ed.). Rio de Janeiro: Ed. Fiocruz.

32. Da Ros, M. A. (2000). Estilos de pensamento em saúde pública: um estudo da produção da FSP USP e ENSP - FIOCRUZ, entre 1948 e 1994, a partir da epistemologia de Ludwick Fleck. (Tese Doutorado em Educação). Programa de Pós-Graduação em Educação, Universidade Federal de Santa Catarina, Florianópolis, SC.

33. Sícoli, J. L., \& Nascimento, P. R. (2003). Promoção de saúde: concepções, princípios e operacionalização. Interface - Comunicação, Saúde, Educação, 7(12), 91-112.

34. WENDHAUSEN, Agueda; SAUPE, Rosita. Concepções de educação em saúde e a estratégia de saúde da família. Texto Contexto de Enfermagem, Florianópolis, v. 12, n. 1, p. 17-25, jan./mar. 2003.

35. Matos, E., Gonçalves, J. R., \& Ramos, F. R. S. (2005). A epistemologia de Ludwick Fleck: subsídios para a prática interdisciplinar em saúde. Texto Contexto - Enferm. (online), 14(3), 383390. doi: 10.1590/S0104-7072005000300009. 
36. OLIVEIRA, S.R.G.; WENDHAUSEN, A.L.P. (RE)SIGNIFICANDO A EDUCAÇÃO EM SAÚDE: DIFICULDADES E POSSIBILIDADES DA ESTRATÉGIA SAÚDE DA FAMÍLIA. Trab. Educ. Saúde, Rio de Janeiro, v. 12 n. 1, p. 129-147, jan./abr. 2014

37. MIRANDA, Karla C. L.; BARROSO, Maria G. T. A contribuição de Paulo Freire à prática da educação crítica em enfermagem. Revista Latino-Americana de Enfermagem, Ribeirão Preto, v.12, n. 4, jul./ago.2004.

38. Freire, P. Pedagogia do oprimido. Rio de Janeiro: Paz e Terra, 2005.

39. MAEYAMA, M.A.; CUTOLO, L.R.A. As concepções de saúde e suas ações consequentes. Arquivos Catarinenses de Medicina, Vol. 39, no. 1, p. 89-96, 2010.

40. OPAS. Carta de Ottawa. Ottawa, 1986.

41. Da Ros MA. A ideologia nos cursos de medicina. In: Marins JJN, Rego S, Lampert JB, Araújo JGC (Orgs.). Educação médica em transformação: instrumentos para a construção de novas realidades. São Paulo: Hucitec,2004. p. 224-244.

42. Cutolo, L. R. A. Estilo de Pensamento em Educação Médica - Um estudo do currículo do curso de graduação em Medicina da UFSC. Tese de Doutorado - CED/USFC. Florianópolis, 2001.

43. Ceccim, R.B. Pacientes impacientes: Paulo Freire. Brasil. Ministerio da Saude. Secretaria de Gestao Estrategica e Participativa. Departamento de Apoio à Gestao Participativa. Caderno de educacao popular e saude - Brasilia, 2007.160:32-45.

44. Garner P, Panpanich R, Logan S. Is routine grouth monitoring effective? A systematic review of trials. Arch Dis Child 2000;82:197-201.

45. BRASIL. Ministério da Saúde. Secretaria de Vigilância em Saúde. Política nacional de promoção da saúde. Brasília: Ministério da Saúde, 2006.

FLEURY-TEIXEIRA, P.; VAZ, F. A. C.; CAMPOS, F. C. C.; ÁlVARES, J.; AGUIAR, R. A. T.; OLIVEIRA, V. A. Autonomia como categoria central no conceito de promoção de saúde. Ciência e Saúde Coletiva, v. 13, sup. 2, p. 2115-2122, 2008. 
ROTEIRO (Forma impressa - dobrável)

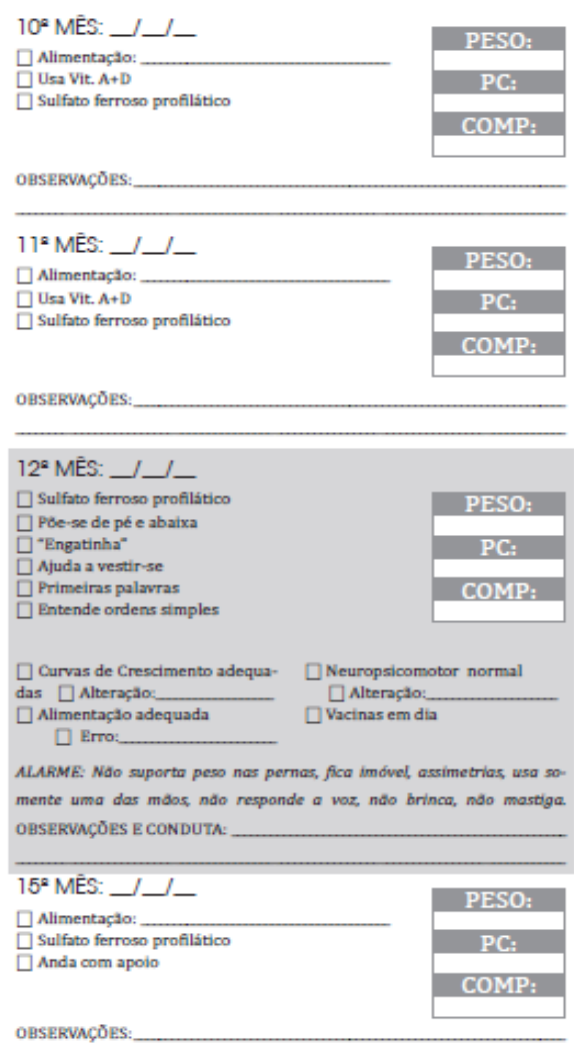

OBSERVAÇÕES:

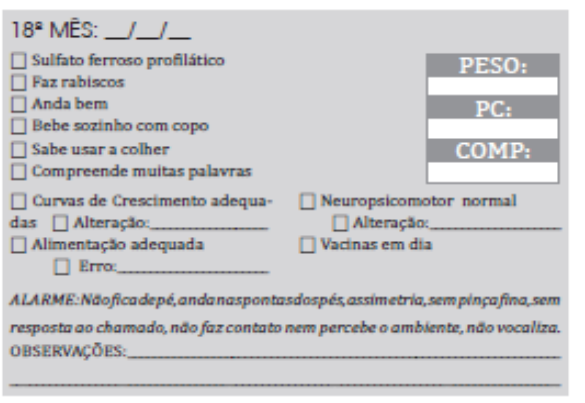

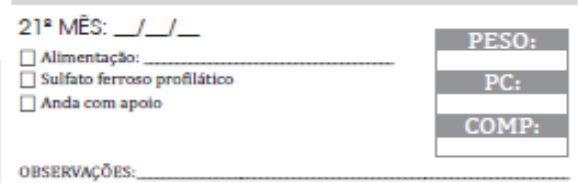

OBSERVŅ্̧E:

\section{ANOS: $-1-$}

$\square$ Sulfato ferroso profilation

$\square$ Aponta partes do corpo

$\square$ Follheia livio

Carre, sobe e desce escadas

$\square$ Abre portas

Usa bem a colher

Coloca o calcs do

$\square$ Curvas de Crescimento adequa- $\quad \square$ Neuropsicomotor normal

$\square$ das $\square$ Alteraçalo: $\square$ Nadinas em dia

$\square$ Erro:

ALARME: Nato anda, ndo constroi nada, ndo compreende o que the dizem, moso pronuncia palovros intelligivels ndo faz contato, estrobismo. öservnçōos:
AvaliaçĀo da CRIANÇA DE BAIXO RISCO

\begin{tabular}{|c|c|}
\hline \multicolumn{2}{|c|}{ 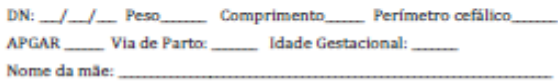 } \\
\hline \multicolumn{2}{|c|}{ 口 Sem intercorrenclass na gravidez/parto } \\
\hline \multicolumn{2}{|l|}{$\square$ Sern intercorrenclias neonatais } \\
\hline \multicolumn{2}{|c|}{$\square$ Exame do reflexo luminoso normal $\square$ alterado } \\
\hline \multicolumn{2}{|c|}{$\square$ Exame de evocaça normal $\square$ alterado } \\
\hline \multicolumn{2}{|l|}{ FATORES DE RISCO: } \\
\hline 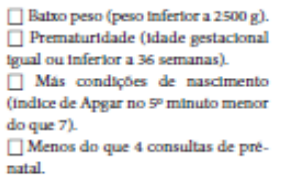 & 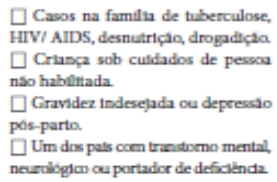 \\
\hline 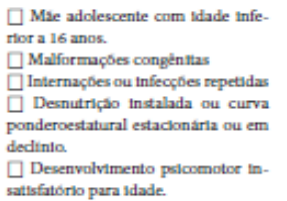 & 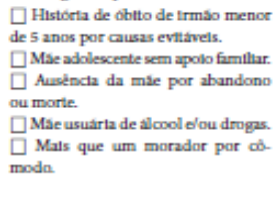 \\
\hline \multicolumn{2}{|l|}{ ORIENTAÇOES: } \\
\hline 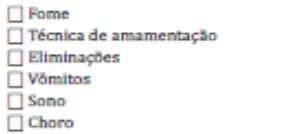 & 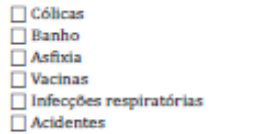 \\
\hline
\end{tabular}

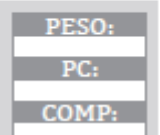

20: Nao firma a cabepa, ndoperceche sans, pabrena de movimentas malo sempre fochada, membros rigidas, mato vo calliza, estrabismo constante, ndo oilha ou segura abjetas OBSERVNCOES E CONDUTA:

\begin{tabular}{|c|c|}
\hline$\square$ Alteraç̧a: & PESOE: \\
\hline $\begin{array}{l}\square \text { Alimentaçso adequada } \\
\square \text { Erro: }\end{array}$ & PC: \\
\hline $\begin{array}{c}\square \text { Neuropsicomotor mormal } \\
\square \text { Aiteraça: }\end{array}$ & COMP: \\
\hline
\end{tabular}

ALARME: NAo sorri, nalosegue a face humana, nunca fica em alerta, ndo inter. age, hipo ou hipertanid dade quando colocado de pe, nab faz forca no pescopo. oBSERVNÇOES E CONDUTA:

\begin{tabular}{|c|c|}
\hline $4^{2} \mathrm{MES}:-1-$ & PESO: \\
\hline $\begin{array}{l}\square \text { MME } \\
\square \text { Fórmula em dilluiçalo ideal } \\
\square \text { Outros }\end{array}$ & PC: \\
\hline $\begin{array}{l}\text { Usa Vit. A+D } \\
\square \text { Rola sem auxilio }\end{array}$ & COMP: \\
\hline
\end{tabular}

OHSERVNCOCES:

5 MÊS: - / -

$\square$ AME

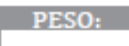

$\square$ Fórmula em dilutçăo ideal

Usa Vit. A+

$\square$ Senta com apoio

ösERVNÇס̄ES:

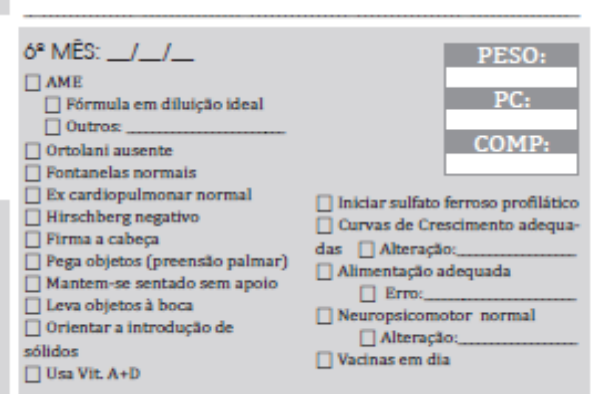

\begin{tabular}{|c|c|}
\hline \multirow{3}{*}{ 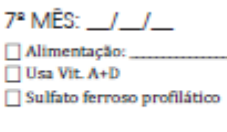 } & PESO: \\
\hline & 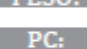 \\
\hline & \\
\hline \multicolumn{2}{|l|}{ OBSERVMØOE:- } \\
\hline \multirow{4}{*}{ 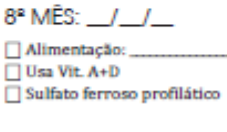 } & PESO. \\
\hline & \\
\hline & PC: \\
\hline & COMP: \\
\hline
\end{tabular}

OBSERVAÇOES:

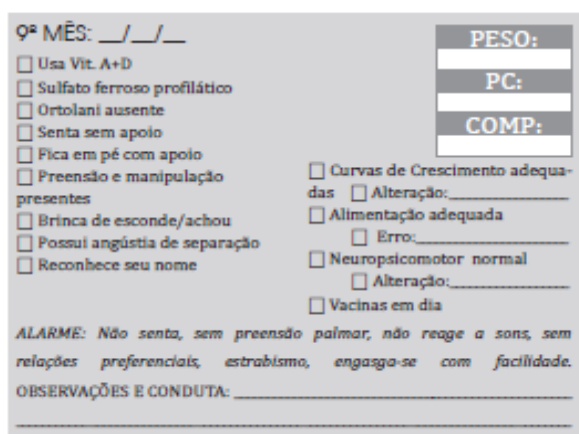

